\title{
The (US) National Lung Cancer Screening Trial: intensifying the debate about the introduction of screening for lung cancer
}

\author{
DR Baldwin \\ Consultant Respiratory Physician, Nottingham University Hospitals and Honorary Associate Professor, University of Nottingham, \\ Respiratory Medicine Unit, David Evans Centre, Nottingham City Hospital Campus, UK
}

TITLE Reduced lung-cancer mortality with low-dose computed tomographic screening.

AUTHORS: National Lung Screening Trial Research Team, Aberle DR, Adams AM et al.

JOURNAL N Engl J Med 20I I; 365:395-409. http://dx.doi.org/I0.1056/ NEJMoa I I 02873

DECLARATION OF INTERESTS Dr Baldwin is the lead respiratory physician on the United Kingdom Lung Cancer Screening (UKLS) project. He has received a consultancy fee from Roche, a grant from the National Institutes of Health Research relating to the UKLS and funding for an educational grant from Pierre Fabre.

\author{
Correspondence to DR Baldwin \\ University of Nottingham \\ Respiratory Medicine Unit, \\ David Evans Centre, Nottingham \\ City Hospital Campus \\ NG5 IPB, UK
}

\author{
tel. $+44(0) / I 5969$ / /69 \\ ext 57462
}

e-mail David.baldwin@nuh.nhs.uk

\section{SUMMARY}

In November 2010 the National Lung Cancer Screening Trial (NLST), a large randomised trial of helical computed tomography (CT) screening for lung cancer versus chest radiograph, was stopped a year early as the required end point of a $20 \%$ reduction in lung cancer mortality had been reached.' This was the first conclusive evidence that was sufficiently free of lead time, length time and over-diagnosis bias, factors that had proven problematic in earlier randomised trials. The NLST enrolled 53,454 subjects aged 55-75 with at least a 30 pack-year smoking history who had quit smoking within the previous 15 years. Between 2000 and 2002 subjects were randomised to receive an annual CT or chest radiograph for three consecutive years. Subjects were found to be well matched to the US census data except that they were younger, better educated and more likely to be former smokers. Compliance with screening was over $90 \%$. There were 247 deaths per 100,000 person years in the CT group compared with 309 deaths per 100,000 person years in the radiography group, representing a $20.0 \%$ reduction $(95 \%$ confidence interval $[\mathrm{Cl}], 6.8$ to 26.7). The all-cause mortality was also reduced by $6.7 \%$ $(95 \% \mathrm{Cl}, 1.2$ to 13.6$)$. In the first two screening rounds, $27 \%$ and $28 \%$ of scans respectively were classified as positive and of these, $96.4 \%$ were false positives. The level of physical harm reported was low and similar in the two arms; psychological harm was not reported.

\section{OPINION}

The NLST has shifted the emphasis of the screening debate from efficacy in reducing mortality to deciding on the applicability of the results to other populations and whether, and in what form, CT screening should be introduced. ${ }^{2}$ If the results can be reproduced in the UK, it is likely that more than 3,000 lives will be saved each year. There remain however uncertainties that make it difficult to design a screening programme without significant financial risk or the prospect of excluding people that may benefit considerably. Key questions concern the selection of patients for screening programmes - should more detailed risk assessment scores be used to avoid screening groups where there is little benefit, as well as allowing a more permissive approach in those at greater risk by virtue of their age? The risk of cancer is much greater in the older age group, yet in the NLST $42.8 \%$ of participants were aged 55-59. Although age-specific cancer rates have not been published, it is likely that this high proportion of younger patients would have considerably diluted the impact of the three annual screens in the early years. Costeffectiveness data have not yet been published on the NLST but there is at least one paper about a model based on the NLST and it shows incremental costeffectiveness ratios (ICERs) well above the National Institute for Health and Clinical Excellence (NICE) thresholds, even when $6 \%$ smoking cessation rates are assumed. ${ }^{3}$ Cost is also a key issue in deciding on the 
optimal frequency and duration of screening. The NLST would suggest that screening should be annual from the age of 55 to 75 but other studies, including the DutchBelgian NELSON trial ${ }^{4}$ and the United Kingdom Lung Screen trial ${ }^{5}$ may clarify this. The optimal management of positive screening tests also needs to be determined to both reduce costs and minimise morbidity. ${ }^{3}$

\section{REFERENCES}

I National Lung Screening Trial Research Team, Aberle DR, Adams $\mathrm{AM}$ et al. Reduced lung-cancer mortality with low-dose computed tomographic screening. N Engl J Med 20I I; 365:395-409. http:// dx.doi.org// 0. I056/NEJMoa I I 02873

2 Field JK, Smith DA, Aberle DR et al. International Association for the Study of Lung Cancer Computed Tomography Screening Workshop 20II Report. J Thorac Oncol. Forthcoming 2012. http:// dx.doi.org/10.1097/JTO.0b0 I 3e31823c58ab

3 McMahon PM, Kong CY, Bouzan C et al. Cost-effectiveness of computed tomography screening for lung cancer in the United States. J Thorac Oncol 20I I; 6:184I-8. http://dx.doi.org/I0.1097/ |TO.0b0I3e31822e59b3
Uncertainties notwithstanding, the NLST has reshaped our thinking about lung cancer screening and although the debate continues about the introduction of screening programmes, we are undoubtedly closer to seeing large lung cancer-specific mortality reductions, a change in the proportion of patients with curable lung cancer presenting to clinics and considerably more work for radiologists and radiographers.

4 van Klaveren RJ, Oudkerk M, Prokop $M$ et al. Management of lung nodules detected by volume CT scanning. N Engl J Med 2009; 36I:222 I-9. http://dx.doi.org/I0.I056/NEJMoa0906085

5 Baldwin DR, Duffy SW, Wald NJ et al. UK Lung Screen (UKLS) nodule management protocol:modelling of a single screen randomised controlled trial of low-dose CT screening for lung cancer. Thorax 20II; 66:308-13. http://dx.doi.org/I0.1 I36/thx.2010.152066

\section{INVITATION TO SUBMIT PAPERS}

We would like to extend an invitation to all readers of The Journal of the Royal College of Physicians of Edinburgh to contribute original material, especially to the clinical section. The JRCPE is a peer-reviewed journal with a circulation of 8,000 . It is also available open access online. Its aim is to publish a range of clinical, educational and historical material of cross-specialty interest to the College's international membership.

The JRCPE is currently indexed in Medline, Embase, Google Scholar and the Directory of Open Access Journals. The editorial team is keen to continue to improve both the quality of content and its relevance to clinical practice for Fellows and Members. All papers are subject to peer review and our turnaround time for a decision averages only eight weeks.

We would be pleased to consider submissions based on original clinical research, including pilot studies. The JRCPE is a particularly good forum for research performed by junior doctors under consultant supervision. We would also consider clinical audits where the 'loop has been closed' and a demonstrable clinical benefit has resulted.

For further information about submissions, please visit: http://www.rcpe.ac.uk/journal/contributers.php or e-mail editorial@rcpe.ac.uk.Thank you for your interest in the College's journal.

The editorial team,

The Journal of the Royal College of Physicians of Edinburgh 